Pacific Journal of Mathematics

DIRECTLY FINITE ALEPH-NOUGHT-CONTINUOUS REGULAR 


\title{
DIRECTLY FINITE ALEPH-NOUGHT-CONTINUOUS REGULAR RINGS
}

\author{
K. R. GOODEARL
}

This paper develops a structure theory for any directly finite, right $\boldsymbol{\aleph}_{0}$-continuous, regular ring $R$, generalizing the corresponding theory for a right and left $\boldsymbol{\aleph}_{0}$-continuous regular ring. The key result is that $R$ must be unit-regular, which also provides a proof, much simpler than previous proofs, that any right and left $\boldsymbol{\aleph}_{0}$-continuous regular ring is unit-regular. It is proved, for example, that $R$ modulo any maximal two-sided ideal is a right self-injective ring. The Grothendieck group $K_{0}(R)$ is shown to be a monotone $\sigma$-complete interpolation group, which leads to an explicit representation of $K_{0}(R)$ as a group of affine continuous realvalued functions on the space of pseudo-rank functions on $R$. It follows, for example, that the isomorphism classes of finitely generated projective right $R$-modules are determined modulo the maximal two-sided ideals of $R$. For another example, if every simple artinian homomorphic image of $R$ is a $t \times t$ matrix ring (for a fixed $t \in N$ ), then $R$ is a $t \times t$ matrix ring.

Introduction. Recall that a regular ring $R$ is said to be right $\boldsymbol{S}_{0}$-continuous provided the lattice $L\left(R_{R}\right)$ of principal right ideals of $R$ is upper $\aleph_{0}$-continuous, i.e., every countable subset of $L\left(R_{R}\right)$ has a supremum in $L\left(R_{R}\right)$, and

$$
A \wedge\left(\bigvee_{n=1}^{\infty} B_{n}\right)=\bigvee_{n=1}^{\infty}\left(A \wedge B_{n}\right)
$$

for every $A \in L\left(R_{R}\right)$ and every countable ascending chain $B_{1} \leqq B_{2} \leqq \cdots$ in $L\left(R_{R}\right)$. Equivalently, $R$ is right $\boldsymbol{\aleph}_{0}$-continuous if and only if every countably generated right ideal of $R$ is essential in a principal right ideal of $R$ [1, Corollary 14.4]. Similarly, $R$ is left $\boldsymbol{\aleph}_{0}$-continuous provided the lattice $L\left({ }_{R} R\right)$ of principal left ideals of $R$ is upper $\aleph_{0}$-continuous, or equivalently, the lattice $L\left(R_{R}\right)$ is lower $\aleph_{0}$-continuous. An $\boldsymbol{\aleph}_{0}$-continuous regular ring is a regular ring that is both right and left $\boldsymbol{\aleph}_{0}$-continuous.

The purpose of this paper is to show that the known structure theory for $\boldsymbol{\aleph}_{0}$-continuous regular rings $[3,6,7]$ carries over nearly intact for directly finite, right $\boldsymbol{\aleph}_{0}$-continuous, regular rings. This may be viewed as a generalization, since all $\boldsymbol{\aleph}_{0}$-continuous regular rings are directly finite [1, Proposition 14.20]. It is an open question whether all directly finite, right $\aleph_{0}$-continuous, regular rings are 
$\aleph_{0}$-continuous, but we conjecture that the answer is negative. For in particular, a positive answer would imply a positive answer to Roos' conjecture that every directly finite, regular, right self-injective ring is left self-injective [1, Problem 14, p. 345], and this conjecture is also suspected to have a negative answer. On the other hand, constructing a counterexample to the $\boldsymbol{\aleph}_{0}$-continuous question might prove easier than constructing a counterexample to Roos' conjecture.

In $\S$ I we prove the key result of the paper, that any directly finite, right $\aleph_{0}$-continuous, regular ring $R$ is unit-regular, along with a few related results. In particular, we show that $R$ modulo any maximal two-sided ideal is a right self-injective ring. In $\S \mathrm{II}$, we investigate the behavior of finitely generated projective right $R$-modules, and study how that behavior is codified in the partially ordered Grothendieck group $K_{0}(R)$. This group is proved to be a monotone $\sigma$-complete interpolation group, and various properties of $R$ follow therefrom. For instance, the isomorphism classes of finitely generated projective right $R$-modules are determined by the isomorphism classes modulo maximal two-sided ideals of $R$, and also determined by the values of extremal pseudo-rank functions of $R$. Section III derives further structural properties of $R$ that follow from countable interpolation, a consequence of the monotone $\sigma$-complete and interpolation properties on $K_{0}(R)$. For example, if every simple artinian homomorphic image of $R$ is a $t \times t$ matrix ring (for some fixed $t \in N$ ), then $R$ is a $t \times t$ matrix ring.

All rings in this paper are associative with 1 , and all modules are unital. We refer the reader to [1] for the general theory of regular rings, and to [1,3] for the general development of $K_{0}$ of regular rings as partially ordered abelian groups, and for the theory of partially ordered abelian groups via their state spaces. We also follow the notation used in these two references. In particular, given modules $A$ and $B$, we write $A \leqq_{8} B$ to mean that $A$ is an essential submodule of $B$ and we write $A \lesssim B$ to mean that $A$ is isomorphic to a submodule of $B$, while we use $n A$ to denote the direct sum of $n$ copies of $A$.

I. Unit-regularity. This section contains our key result, that every directly finite, right $\boldsymbol{\aleph}_{0}$-continuous, regular ring is unit-regular. As an application of this result, we prove that all simple homomorphic images of directly finite, right $\aleph_{0}$-continuous, regular rings are right self-injective. Several related results are also proved.

We shall have need of the maximal right $\aleph_{0}$-quotient ring [1, pp. 177, 178] associated with any right $\boldsymbol{\aleph}_{0}$-continuous regular ring $R$; this is a right quotient ring $S$ of $R$ which is right $\boldsymbol{\aleph}_{0}$-continu- 
ous, regular, and right $\aleph_{0}$-injective, and $R$ contains all the idempotents of $S$ [1, Theorem 14.12]. We shall use this result a number of times, without further explicit reference to it.

The proof of our first proposition has been considerably simplified through the use of successive suggestions by S. K. Berberian and P. Menal, whom we thank for these suggestions.

Proposition 1.1. Let $R$ be a right $\boldsymbol{\aleph}_{0}$-continuous regular ring, and let $S$ be its maximal right $\boldsymbol{\aleph}_{0}$-quotient ring. If $R$ is directly finite, then neither $R$ nor $S$ contains an infinite direct sum of nonzero pairwise isomorphic right ideals. In particular, $S$ is directly finite.

Proof. If $S$ contains no infinite direct sums of nonzero pairwise isomorphic right ideals, then the same is true of $R$, since any infinite independent family $\left\{J_{i}\right\}$ of nonzero pairwise isomorphic right ideals of $R$ would generate an infinite independent family $\left\{J_{i} S\right\}$ of nonzero pairwise isomorphic right ideals of $S$. In addition, this direct sum condition on $S$ implies direct finiteness, by [1, Corollary 5.6]. Thus we need only show that $S$ contains no infinite direct sums of nonzero pairwise isomorphic right ideals.

It suffices to show that if $\left\{A_{1}, A_{2}, \cdots\right\}$ is an independent sequence of pairwise isomorphic principal right ideals of $S$, then $A_{1}=0$.

Since $S$ is right $\aleph_{0}$-continuous, it contains principal right ideals $B$ and $C$ such that

$$
\bigoplus_{n=1}^{\infty} A_{2 n} \leqq_{e} B \text { and } \bigoplus_{n=0}^{\infty} A_{2 n+1} \leqq_{e} C
$$

Note that $B \cap C=0$, and that

$$
\bigoplus_{n=1}^{\infty} A_{n} \leqq_{e} B \oplus C
$$

Choose an idempotent $e \in S$ such that $e S=B \oplus C$. There is an isomorphism

$$
\bigoplus_{n=1}^{\infty} A_{2 n} \longrightarrow \bigoplus_{n=0}^{\infty} A_{2 n+1}
$$

which must, because $S$ is right $\boldsymbol{\aleph}_{0}$-injective, be given by left multiplication by an element $x \in S$. Inasmuch as $\bigoplus A_{2 n} \leqq_{e} B$ and $\oplus A_{2 n+1} \leqq_{e} C$, and $S$ is a right nonsingular ring, it follows that $x$ induces an isomorphism of $B$ onto $C$. Similarly, $B \cong e S$, and thus $e S \cong e S \oplus e S$.

In particular, the ring $e S e$ is isomorphic to the $2 \times 2$ matrix ring $M_{2}(e S e)$, from which we infer that $e S e$ is generated (as a ring) by its idempotents. Since all the idempotents in $S$ actually lie in 
$R$, we thus obtain $e \in R$ and $e S e=e R e$.

The direct finiteness of $R$ implies that $e R e$ is a directly finite ring. Thus $e S e$ is a directly finite ring, hence $e S$ is a directly finite module. As $e S \cong e S \oplus e S$, we conclude that $e S=0$. Therefore $A_{1}=0$, as desired.

The following easy lemma appears in [2, Lemma 2.2]. We include its short proof for the sake of completeness.

Lemma 1.2. Let $A, B, C$ be finitely generated projective right modules over a regular ring, such that $A \oplus C \cong B \oplus C$. Then there exist decompositions

$$
A=A_{1} \oplus A_{2} ; \quad B=B_{1} \oplus B_{2} ; \quad C=C_{1} \oplus C_{2}
$$

such that $A_{1} \cong B_{1}$ and $A_{2} \oplus C_{2} \cong B_{2} \oplus C_{2}$, while also $A_{2} \cong C_{1}$.

Proof. Using [1, Theorem 2.8], we obtain decompositions $A=$ $A_{1} \oplus A_{2}$ and $C=D \oplus E$ such that $A_{1} \oplus D \cong B$ and $A_{2} \oplus E \cong C$. Consequently, there are decompositions $B=B_{1} \oplus B_{2}$ and $C=C_{1} \oplus C_{2}$ such that $B_{1} \cong A_{1}$ and $B_{2} \cong D$, while also $C_{1} \cong A_{2}$ and $C_{2} \cong E$. Finally,

$$
A_{2} \oplus C_{2} \cong C_{1} \oplus C_{2}=C=D \oplus E \cong B_{2} \oplus C_{2} \text {. }
$$

Lemma 1.3. Let $A, B, C$ be finitely generated projective right modules over a regular ring, such that $A \oplus C \cong B \oplus C$. Then there exist decompositions

$$
A=A^{\prime} \oplus A^{\prime \prime} ; \quad B=B^{\prime} \oplus B^{\prime \prime} ; \quad C=C^{\prime} \oplus C^{\prime \prime}
$$

such that $A^{\prime} \cong B^{\prime}$ and $A^{\prime \prime} \oplus C^{\prime \prime} \cong B^{\prime \prime} \oplus C^{\prime \prime}$, while also $A^{\prime \prime} \oplus B^{\prime \prime} \lesssim C^{\prime}$.

Proof. We start with the decompositions given by Lemma 1.2. Since $B_{2} \oplus C_{2} \cong A_{2} \oplus C_{2}$, a second application of Lemma 1.2 yields decompositions

$$
B_{2}=B_{3} \oplus B^{\prime \prime} ; \quad A_{2}=A_{3} \oplus A^{\prime \prime} ; \quad C_{2}=C_{3} \oplus C^{\prime \prime}
$$

such that $B_{3} \cong A_{3}$ and $B^{\prime \prime} \oplus C^{\prime \prime} \cong A^{\prime \prime} \oplus C^{\prime \prime}$, while also $B^{\prime \prime} \cong C_{3}$. Now set

$$
A^{\prime}=A_{1} \oplus A_{3} ; \quad B^{\prime}=B_{1} \oplus B_{3} ; \quad C^{\prime}=C_{1} \oplus C_{3}
$$

so that we have decompositions

$$
A=A^{\prime} \oplus A^{\prime \prime} ; \quad B=B^{\prime} \oplus B^{\prime \prime} ; \quad C=C^{\prime} \oplus C^{\prime \prime}
$$

with $A^{\prime} \cong B^{\prime}$. In addition, 


$$
A^{\prime \prime} \oplus B^{\prime \prime} \leqq A_{2} \oplus B^{\prime \prime} \cong C_{1} \oplus C_{3}=C^{\prime} \text {. }
$$

We are now in position to prove our unit-regularity theorem. The method is a modification of that used to prove that all $N^{*}$ complete regular rings are unit-regular, in [2, Theorem 2.3].

THEOREM 1.4. If $R$ is a directly finite, right $\boldsymbol{\aleph}_{0}$-continuous, regular ring, then $R$ is unit-regular.

Proof. According to [1, Theorem 4.1], it suffices to show that if $A, B, C$ are any principal right ideals of $R$ satisfying $A \oplus C \cong$ $B \oplus C$, then $A \cong B$. Using Lemma 1.3 , we obtain decompositions

$$
A=A^{\prime} \oplus A^{\prime \prime} ; \quad B=B^{\prime} \oplus B^{\prime \prime} ; \quad C=C^{\prime} \oplus C^{\prime \prime}
$$

such that $A^{\prime} \cong B^{\prime}$ and $A^{\prime \prime} \oplus C^{\prime \prime} \cong B^{\prime \prime} \oplus C^{\prime \prime}$, while also $A^{\prime \prime} \oplus B^{\prime \prime} \lesssim C^{\prime}$. In particular, $A^{\prime \prime} \oplus B^{\prime \prime} \lesssim R_{R}$. Since it suffices to prove that $A^{\prime \prime} \cong$ $B^{\prime \prime}$, we may replace $A, B, C$ by $A^{\prime \prime}, B^{\prime \prime}, C^{\prime \prime}$. Thus we may assume, without loss of generality, that $A \cap B=0$.

Now apply Lemma 1.3 infinitely many times in succession, obtaining principal right ideals

$$
\begin{aligned}
& A_{1}^{\prime}, A_{1}^{\prime \prime}, A_{2}^{\prime}, A_{2}^{\prime \prime}, \cdots \leqq A ; \quad B_{1}^{\prime}, B_{1}^{\prime \prime}, B_{2}^{\prime}, B_{2}^{\prime \prime}, \cdots \leqq B ; \\
& C_{1}^{\prime}, C_{1}^{\prime \prime}, C_{2}^{\prime}, C_{2}^{\prime \prime}, \cdots \leqq C
\end{aligned}
$$

such that

$$
A=A_{1}^{\prime} \oplus A_{1}^{\prime \prime} ; \quad B=B_{1}^{\prime} \oplus B_{1}^{\prime \prime} ; \quad C=C_{1}^{\prime} \oplus C_{1}^{\prime \prime}
$$

while also

$$
\begin{aligned}
& A_{n}^{\prime \prime}=A_{n+1}^{\prime} \oplus A_{n+1}^{\prime \prime} ; \quad B_{n}^{\prime \prime}=B_{n+1}^{\prime} \oplus B_{n+1}^{\prime \prime} ; \quad C_{n}^{\prime \prime}=C_{n+1}^{\prime} \oplus C_{n+1}^{\prime \prime} \\
& A_{n}^{\prime} \cong B_{n}^{\prime} ; \quad A_{n}^{\prime \prime} \oplus C_{n}^{\prime \prime} \cong B_{n}^{\prime \prime} \oplus C_{n}^{\prime \prime} ; \quad A_{n}^{\prime \prime} \oplus B_{n}^{\prime \prime} \lesssim C_{n}^{\prime}
\end{aligned}
$$

for all $n$. Note that $\left\{A_{1}^{\prime}, A_{2}^{\prime}, \cdots\right\}$ and $\left\{B_{1}^{\prime}, B_{2}^{\prime}, \cdots\right\}$ are independent sequences of right ideals. Since $A \cap B=0$, it follows that the sequence $\left\{A_{1}^{\prime}, B_{1}^{\prime}, A_{2}^{\prime}, B_{2}^{\prime}, \cdots\right\}$ is independent as well.

We claim that $\oplus A_{n}^{\prime} \leqq_{e} A$ and $\oplus B_{n}^{\prime} \leqq_{e} B$. Thus consider any right ideal $E \leqq A$ such that $E \cap\left(\oplus A_{n}^{\prime}\right)=0$. For each $n$, we have

$$
A=A_{1}^{\prime} \oplus A_{2}^{\prime} \oplus \cdots \oplus A_{n}^{\prime} \oplus A_{n}^{\prime \prime} \text { and } E \cap\left(A_{1}^{\prime} \oplus A_{2}^{\prime} \oplus \cdots \oplus A_{n}^{\prime}\right)=0 \text {, }
$$

whence $E \lesssim A_{n}^{\prime \prime} \lesssim C_{n}^{\prime}$. As $\left\{C_{1}^{\prime}, C_{2}^{\prime}, \cdots\right\}$ is an independent sequence of right ideals of $R$, it follows that

$$
\aleph_{0} E \lesssim C_{1}^{\prime} \oplus C_{2}^{\prime} \oplus \cdots \leqq R_{R}
$$

However, $R$ contains no infinite direct sums of nonzero pairwise 
isomorphic right ideals, by Proposition 1.1, hence we must have $E=0$. Thus $\oplus A_{n}^{\prime} \leqq_{e} A$, and similarly $\oplus B_{n}^{\prime} \leqq_{e} B$, as claimed.

In the lattice $L\left(R_{R}\right)$, we now have $A=\vee A_{n}^{\prime}$ and $B=\vee B_{n}^{\prime}$, while also $A_{n}^{\prime} \cong B_{n}^{\prime}$ for all $n$. As the sequence $\left\{A_{1}^{\prime}, B_{1}^{\prime}, A_{2}^{\prime}, B_{2}^{\prime}, \cdots\right\}$ is independent, [1, Lemma 14.22] shows that $A \cong B$, as desired.

Theorem 1.4 provides an affirmative answer to [1, Problem 23, p. 346].

CoROLlaRY 1.5. Let $R$ be a right $\boldsymbol{\aleph}_{0}$-continuous regular ring, and let $S$ be its maximal right $\boldsymbol{\aleph}_{0}$-quotient ring. If $R$ is directly finite, then $R$ and $S$ are both unit-regular.

Proof. Proposition 1.1 and Theorem 1.4.

Corollary 1.6. [6, Theorem 3.2] Every $\aleph_{0}$-continuous regular ring is unit-regular.

Proof. Every $\boldsymbol{\aleph}_{0}$-continuous regular ring is directly finite by [1, Proposition 14.20], so Theorem 1.4 applies.

A direct proof of Corollary 1.6 alone can be obtained as follows. First prove that $\boldsymbol{\aleph}_{0}$-continuous regular rings contain no infinite direct sums of nonzero pairwise isomorphic right ideals [1, Proposition 14.20], then prove Lemmas 1.2 and 1.3, and finally, use the proof of Theorem 1.4.

Handelman's original proof in [6] of the unit-regularity of an $\aleph_{0}$-continuous regular ring $R$ required (i) the development of the maximal $\aleph_{0}$-quotient ring $S$ of $R$ to the point where it could be shown that $M_{2}(S)$ is $\aleph_{0}$-continuous; (ii) a lattice-theoretic theorem of Halperin [4, Theorem 5.1], that perspectivity is always transitive in an $\boldsymbol{\aleph}_{0}$-continuous geometry; (iii) another theorem of Handelman, that a regular ring $S$ is unit-regular if and only if perspectivity is transitive in the lattice of principal right ideals of $M_{2}(S)$ [5, Theorem 15]; and (iv) a final short argument to transfer unit-regularity from $S$ to $R$. This method of proof is quite long and complicated. A somewhat shorter proof, basically a module-theoretic version of Halperin's transitivity argument, appeared in [1, Theorem 14.24]. This method is still somewhat convoluted. Thus we may tout our present proof as an answer to Handelman's plea for a short proof of this result [6, p. 189].

For a right $\boldsymbol{\aleph}_{0}$-continuous regular ring $R$, left $\boldsymbol{\aleph}_{0}$-continuity is sufficient to imply direct finiteness and unit-regularity, as we have seen above. We now show that left $\boldsymbol{\aleph}_{0}$-injectivity, which is some- 
times easier to check, is also sufficient. This answers [1, Problem 24 , p. 346] in the affirmative. We thank $P$. Menal for a suggestion which simplified our original proof.

Lemma 1.7. [Menal] Let $R$ be a left $\boldsymbol{\aleph}_{0}$-injective regular ring, and let $\left\{J_{0}, J_{1}, J_{2}, \cdots\right\}$ be an independent sequence of principal left ideals of $R$. Then there exist orthogonal idempotents $f_{0}, f_{1}, f_{2}, \cdots$ in $R$ such that $R f_{n}=J_{n}$ for all $n$.

Proof. For $k=0,1,2, \cdots$, choose an idempotent $e_{k} \in R$ such that $R e_{k}=J_{k}$. As $R$ is left $\boldsymbol{\aleph}_{0}$-injective, the natural projection $\bigoplus J_{n} \rightarrow J_{k}$ must be given by right multiplication by an element $x_{k} \in R$. Thus $e_{k} x_{k}=e_{k}$ and $e_{n} x_{k}=0$ for all $n \neq k$.

Set $f_{k}=x_{k} e_{k}$ for all $k$, and note that these $f_{k}$ are orthogonal idempotents in $R$. Since $f_{k} e_{k}=f_{k}$ and $e_{k} f_{k}=e_{k}$, we also have $J_{k}=$ $R e_{k}=R f_{k}$ for all $k$.

THEOREM 1.8. Let $R$ be a right $\boldsymbol{\aleph}_{0}$-continuous regular ring. If $R$ is left $\boldsymbol{\aleph}_{0}$-injective, then $R$ is also left $\boldsymbol{\aleph}_{0}$-continuous, and so is unit-regular.

Proof. To check left $\aleph_{0}$-continuity, we must show that any countably generated left ideal $J$ of $R$ is essential in a principal left ideal of $R$. Once this is established, unit-regularity follows from Corollary 1.6.

According to [1, Proposition 2.14], there exist orthogonal idempotents $e_{1}, e_{2}, \cdots$ in $R$ such that $\oplus R e_{n}=J$. As $R$ is right $\boldsymbol{\aleph}_{0}$-continuous, the countably generated right ideal $\oplus e_{n} R$ must be essential in a principal right ideal $A$. Choose an idempotent $e \in R$ such that $e R=A$, set $e_{0}=1-e$, and note that

$$
\bigoplus_{n=0}^{\infty} e_{n} R \leqq(1-e) R \oplus A=R_{R}
$$

Since $e_{0} e_{n}=0$ for all $n=1,2, \cdots$, we see that $R e_{0} \cap J=0$, so that the principal left ideals $R e_{0}, R e_{1}, R e_{2}, \cdots$ are independent. By Lemma 1.7, there exist orthogonal idempotents $f_{0}, f_{1}, f_{2}, \cdots$ in $R$ such that $R f_{n}=R e_{n}$ for all $n$, and we claim that $\oplus R f_{n} \leqq_{R} R$.

If not, then $R p \cap\left(\oplus R f_{n}\right)=0$ for some nonzero element $p \in R$. Using left $\aleph_{0}$-injectivity again, the natural projection of $R p \oplus\left(\oplus R f_{n}\right)$ onto $R p$ must be given by right multiplication by some element $q \in R$, so that $p q=p$ and $f_{n} q=0$ for all $n$. In particular, $R e_{n} q=$ $R f_{n} q=0$ and so $e_{n} q=0$ for all $n$. Also, $p q \neq 0$, whence $q \neq 0$. Since $\bigoplus e_{n} R \leqq_{e} R_{R}$, there is some $r \in R$ such that $q r \neq 0$ and $q r \in$ $\oplus e_{n} R$. But as $e_{n} q r=0$ for all $n$, this is impossible. 
Thus $\oplus R f_{n} \leqq_{e} R$, as claimed. Since

$$
J=\bigoplus_{n=1}^{\infty} R e_{n}=\bigoplus_{n=1}^{\infty} R f_{n},
$$

we conclude that $J \leqq_{e} R\left(1-f_{0}\right)$. Therefore $J$ is essential in a principal left ideal, as desired.

Returning to directly finite, right $\boldsymbol{\aleph}_{0}$-continuous, regular rings, we wish to prove appropriate one-sided analogs of the structural results known for $\boldsymbol{\aleph}_{0}$-continuous regular rings. The key missing ingredient in such a program was unit-regularity; having proved Theorem 1.4, we can proceed without any major new difficulties. For example, we have the following theorem, which generalizes [6, Corollary 3.2]. Other analogs and generalizations will appear in $\S \S$ II and III.

THeOREM 1.9. Let $R$ be a directly finite, right $\boldsymbol{\aleph}_{0}$-continuous, regular ring, and let $M$ be a maximal two-sided ideal of $R$. Then $R / M$. is a simple, unit-regular, right self-injective ring.

Proof. Since $R$ is unit-regular by Theorem 1.4 , so is $R / M$. In particular, all the matrix rings $M_{n}\left(R / M_{1}\right)$ are directly finite, hence [1, Proposition 5.9] shows that $R / M$. contains no uncountable direct sums of nonzero right ideals. Then $R / M$ must be a right continuous regular ring, by [1, Corollary 14.16]. As $R / M$ is indecomposable, we conclude from [1, Corollary 13.20] that $R / M$ is actually right self-injective.

We conclude this section by showing that the argument used in Theorem 1.4 also yields the following unit-regularity result.

THEOREM 1.10. Let $R$ be a regular, right $\aleph_{0}$-injective ring. If $R$ contains no infinite direct sums of nonzero pairwise isomorphic right ideals, then $R$ is unit-regular.

Proof. By [1, Theorem 4.1], we need only show that if $A, B, C$ are any principal right ideals of $R$ satisfying $A \oplus C \cong B \oplus C$, then $A \cong B$. As in Theorem 1.4, we obtain independent sequences of cyclic submodules

$$
A_{1}^{\prime}, A_{2}^{\prime}, \cdots \leqq A ; \quad B_{1}^{\prime}, B_{2}^{\prime}, \cdots \leqq B
$$

such that $\bigoplus A_{n}^{\prime} \leqq_{e} A$ and $\bigoplus B_{n}^{\prime} \leqq_{e} B$, while also $A_{n}^{\prime} \cong B_{n}^{\prime}$ for all $n$. Then there is an isomorphism of $\oplus A_{n}^{\prime}$ onto $\bigoplus B_{n}^{\prime}$, which, by right $\boldsymbol{\aleph}_{0}$-injectivity, must be given by left multiplication by some element 
$x \in R$. As $R_{R}$ is nonsingular, we conclude that $x$ induces an isomorphism of $A$ onto $B$.

In view of Theorem 1.10, the following question arises: Is every directly finite, regular, right $\boldsymbol{\aleph}_{0}$-injective ring necessarily unit-regular? Of course, it is well-known that all directly finite, regular, right self-injective rings are unit-regular [1, Theorem 9.17].

II. Projective modules and $K_{0}$. We continue our study of the structure of a directly finite, right $\boldsymbol{\aleph}_{0}$-continuous, regular ring $R$ by investigating the behavior of finitely generated projective right $R$-modules. Much of this behavior is codified in the partially ordered Grothendieck group $K_{0}(R)$, which turns out to be a monotone $\sigma$-complete interpolation group, and much information can be gleaned just from this property. As most of our proofs rely on the unit-regularity of $R$ (Theorem 1.4), we shall not explicitly refer to this theorem each time we use it. Since some of our proofs require $\boldsymbol{\aleph}_{0}$-injectivity at certain stages, we need to be able to transfer problems to the maximal right $\boldsymbol{\aleph}_{0}$-quotient ring of $R$. The following proposition provides a key step in such transfers. It may be viewed as answering the directly finite case of [1, Problem 25, p. 347] affirmatively.

Proposition 2.1. Let $R$ be a directly finite, right $\aleph_{0}$-continuous, regular ring, let $S$ be the maximal right $\mathbf{\aleph}_{0}$-quotient ring of $R$, and let $A$ and $B$ be finitely generated projective right $R$-modules.

(a) If $A \bigotimes_{R} S \lesssim B \bigotimes_{R} S$, then $A \lesssim B$.

(b) If $A \bigotimes_{R} S \cong B \bigotimes_{R} S$, then $A \cong B$.

Proof. Since $S$ is unit-regular and $R$ contains all the idempotents of $S$, these results follow from [1, Proposition 3.16].

THEOREm 2.2. Let $R$ be a directly finite, right $\boldsymbol{\aleph}_{0}$-continuous, regular ring, and let $A$ and $B$ be finitely generated projective right $R$-modules. Let

$$
A_{1}, A_{2}, \cdots \leqq A ; \quad B_{1}, B_{2}, \cdots \leqq B
$$

be independent sequences of finitely generated submodules of $A$ and $B$, such that $A_{n} \cong B_{n}$ for all $n$.

(a) If $\oplus A_{n} \leqq_{e} A$, then $A \lesssim B$.

(b) If $\oplus A_{n} \leqq_{e} A$ and $\oplus B_{n} \leqq_{e} B$, then $A \cong B$.

Proof. Let $S$ denote the maximal right $\boldsymbol{\aleph}_{0}$-quotient ring of $R$, and recall from Proposition 1.1 that $S$ is directly finite. Then 
$A \bigotimes_{R} S$ and $B \bigotimes_{R} S$ are finitely generated projective right $S$-modules, we have independent sequences of finitely generated submodules of $A \otimes_{R} S$ and $B \otimes_{R} S$ that may be labelled

$$
A_{1} \bigotimes_{R} S, A_{2} \bigotimes_{R} S, \cdots ; \quad B_{1} \bigotimes_{R} S, B_{2} \bigotimes_{R} S, \cdots
$$

and $A_{n} \bigotimes_{R} S \cong B_{n} \bigotimes_{R} S$ for all $n$. In case (a), we have

$$
\bigoplus\left(A_{n} \underset{R}{\bigotimes_{R}} S\right) \leqq_{e} A \underset{R}{\bigotimes_{R} S}
$$

and by Proposition 2.1 it suffices to prove that $A \otimes_{R} S \lesssim B \bigotimes_{R} S$; similarly in case (b). Thus there is no loss of generality in assuming that $R$ is right $\boldsymbol{\aleph}_{0}$-injective.

As a consequence, all matrix rings over $R$ are right $\aleph_{0}$-injective and right $\aleph_{0}$-continuous [1, Proposition 14.19]. Using the standard Morita-equivalences, we may transfer our problems to the category of right modules over any matrix ring $M_{k}(R)$. By choosing $k$ large enough, we may arrange for the new modules corresponding to $A$ and $B$ to be cyclic, hence isomorphic to principal right ideals of $M_{k}(R)$.

Therefore we may now assume, without loss of generality, that $A$ and $B$ are principal right ideals of $R$.

(a) There is an isomorphism $\oplus A_{n} \rightarrow \bigoplus B_{n}$, which by right $\aleph_{0}$-injectivity extends to an endomorphism $f$ of $R_{R}$. Since $\oplus A_{n} \leqq_{e} A$, we see that $A \cap \operatorname{ker}(f)=0$. On the other hand, $R / B$ is isomorphic to a direct summand of the nonsingular module $R_{R}$ and so is nonsingular itself, from which we infer that $f(A) \leqq B$. Thus $f$ restricts to a monomorphism of $A$ into $B$.

(b) As in (a), an isomorphism $\oplus A_{n} \rightarrow \bigoplus B_{n}$ extends to a monomorphism $g: A \rightarrow B$. Note that $g(A)$ contains $\oplus B_{n}$ and so is essential in $B$. On the other hand, $g(A)$ is a principal right ideal of $R$ and so is a direct summand of $B$. Therefore $g(A)=B$, whence $A \cong B$.

Theorem 2.2 and the following corollary generalize [1, Corollaries 14.25(a), 14.26, 14.27(a)]. In addition, Theorem 2.2 provides an affirmative answer for the directly finite case of [1, Problem 26, p. 347].

CoRollary 2.3. Let $R$ be a directly finite, right $\boldsymbol{\aleph}_{0}$-continuous, regular ring, and let $A$ and $B$ be finitely generated projective right $R$-modules.

(a) If $A$ has ian ascending sequence $A_{1} \leqq A_{2} \leqq \cdots$ of finitely generated submodules such that $\cup A_{n} \leqq_{e} A$ and $A_{n} \lesssim B$ for all $n$, then $A \lesssim B$.

(b) If there are ascending sequences 


$$
A_{1} \leqq A_{2} \leqq \cdots \leqq A ; \quad B_{1} \leqq B_{2} \leqq \cdots \leqq B
$$

of finitely generated submodules of $A$ and $B$ such that $\cup A_{n} \leqq_{e} A$ and $\cup B_{n} \leqq_{e} B$, while also $A_{n} \cong B_{n}$ for all $n$, then $A \cong B$.

Proof. (a) As $R$ is unit-regular, [1, Corollary 4.10] shows that $\cup A_{n} \lesssim B$. Since $\cup A_{n}$ can be expressed as a countable direct sum of finitely generated submodules, we may apply Theorem 2.2(a).

(b) In view of (a), we have $A \lesssim B$ and $B \lesssim A$. By unit-regularity, it follows that $A \lesssim B$.

Definition. Recall that the Grothendieck group $K_{0}(R)$ of a ring $R$ is an abelian group with generating set

$\{[A] \mid A$ is a finitely generated projective right $R$-module $\}$

and with relations $[A]+[B]=[C]$ whenever $A \oplus B \cong C$. All elements of $K_{0}(R)$ have the form $[A]-[B]$, for suitable $A$ and $B$. We set

$$
K_{0}(R)^{+}=\{[A] \mid A \text { is a finitely generated projective right } R \text {-module }\},
$$

and we define a relation $\leqq$ on $K_{0}(R)$ so that $x \leqq y$ if and only if $y-x$ lies in $K_{0}(R)^{+}$. This relation is a translation-invariant preorder on $K_{0}(R)$, on that $K_{0}(R)$ becomes a pre-ordered abelian group. The element $[R]$ is an order-unit for $K_{0}(R)$, meaning that for any $x \in K_{0}(R)$ there exists $n \in N$ such that $x \leqq n[R]$.

Working with $K_{0}(R)$ is much cleaner in the case of a unitregular ring $R$. In this case, for any finitely generated projective right $R$-modules $A, B, C, D$, we have

$$
\begin{aligned}
& {[A]-[B]=[C]-[D] \text { in } K_{0}(R) \text { if and only if } A \oplus D \cong B \oplus C} \\
& {[A]-[B] \leqq[C]-[D] \text { in } K_{0}(R) \text { if and only if } A \oplus D \lesssim B \oplus C}
\end{aligned}
$$

[1, Proposition 15.2]. In particular, it follows that the relation $\leqq$ on $K_{0}(R)$ is actually a partial order, so that $K_{0}(R)$ is a partially ordered abelian group in this case.

Proposition 2.4. Let $R$ be a directly finite, right $\boldsymbol{\aleph}_{0}$-continuous, regular ring, and let $S$ be the maximal right $\boldsymbol{\aleph}_{0}$-quotient ring of $R$. Then the natural map $K_{0}(R) \rightarrow K_{0}(S)$ (induced by the inclusion map $R \rightarrow S$ ) is an isomorphism of partially ordered abelian groups.

Proof. Let $j: K_{0}(R) \rightarrow K_{0}(S)$ be the natural map, which sends [A] to $\left[A \otimes_{R} S\right.$ ], for any finitely generated projective right $R$-module 
A. It is clear that $j$ is a group homomorphism, and a positive map.

Given any $y \in K_{0}(S)^{+}$, we have $y=[C]$ for some finitely generated projective right $S$-module $C$. Then $C \cong e_{1} S \oplus \cdots \oplus e_{n} S$ for some idempotents $e_{1}, \cdots, e_{n} \in S$. Since each $e_{\imath}$ actually lies in $R$, we can construct a finitely generated projective right $R$-module $A=$ $e_{1} R \oplus \cdots \oplus e_{n} R$ such that $A \otimes_{R} S \cong C$. Defining $x=[A]$, we obtain an element $x \in K_{0}(R)^{+}$such that $j(x)=y$.

Therefore $j\left(K_{0}(R)^{+}\right)=K_{0}(S)^{+}$. In particular, since every element of $K_{0}(S)$ is a difference of two elements from $K_{0}(S)^{++}$, it follows that $j$ is surjective.

Finally, consider any $x \in \operatorname{ker}(j)$, and write $x=[A]-[B]$ for some finitely generated projective right $R$-modules $A$ and $B$. Then

$$
\left[A \bigotimes_{R} S\right]-\left[B \bigotimes_{R} S\right]=j(x)=0 .
$$

Since $S$ is unit-regular, it follows that $A \otimes_{R} S \cong B \otimes_{R} S$, and then Proposition 2.1 shows that $A \cong B$, whence $x=0$.

Thus $j$ is a group isomorphism. Since $j\left(K_{0}(R)^{+}\right)=K_{0}(S)^{+}$, we conclude that $j^{-1}$ is a positive map, hence $j$ is an isomorphism of partially ordered abelian groups.

\section{Proposition 2.4 generalizes [7, Proposition 2.1(i)].}

DEFINITION. A partially ordered abelian group $K$ is called an interpolation group provided the following property holds: given $x_{1}, x_{2}, y_{1}, y_{2}$ in $K$ satisfying $x_{i} \leqq y_{j}$ for all $i, j$, there must exist $z \in K$ such that $x_{i} \leqq z \leqq y_{j}$ for all $i, j$. We say that $K$ is monotone $\sigma$-complete if every ascending sequence $x_{1} \leqq x_{2} \leqq \cdots$ in $K$ that is bounded above has a supremum in $K$.

Much of the structure theory for $\boldsymbol{\aleph}_{0}$-continuous regular rings can be derived from the fact that $K_{0}$ of any such ring is a monotone $\sigma$-complete interpolation group [7, Proposition 2.1(ii)]. This also holds in our context, as follows.

THEOREM 2.5. If $R$ is a directly finite, right $\mathbf{\aleph}_{0}$-continuous, regular ring, then $K_{0}(R)$ is a monotone $\sigma$-complete interpolation group.

Proof. In view of Proposition 2.4, there is no loss of generality in assuming that $R$ is right $\boldsymbol{\aleph}_{0}$-injective. Thus all matrix rings over $R$ are right $\boldsymbol{\aleph}_{0}$-continuous [1, Proposition 14.19].

The unit-regularity of $R$ implies that $K_{0}(R)$ is an interpolation group [3, Proposition II.10.3].

Now consider an ascending sequence $x_{1} \leqq x_{2} \leqq \cdots$ in $K_{0}(R)$ which 
is bounded above. Choose $a \in K_{0}(R)$ such that $a+x_{1} \geqq 0$, so that we obtain a new ascending sequence

$$
0 \leqq a+x_{1} \leqq a+x_{2} \leqq \cdots
$$

in $K_{0}(R)^{+}$which is bounded above. It suffices to find a supremum $b$ for the sequence $\left\{a+x_{n}\right\}$, since then $b-a$ will be a supremum for the sequence $\left\{x_{n}\right\}$. Thus we may assume, without loss of generality, that all $x_{n} \geqq 0$.

Consequently, each $x_{n}=\left[A_{n}\right]$ for some finitely generated projective right $R$-module $A_{n}$, and $A_{1} \lesssim A_{2} \lesssim \cdots$. Since the sequence $\left\{x_{n}\right\}$ is bounded above, there exists $t \in N$ such that all $x_{n} \leqq t[R]$, whence $A_{n} \lesssim t R_{R}$ for all $n$. According to [1, Corollary 4.11], $t R_{R}$ has submodules $B_{1} \leqq B_{2} \leqq \cdots$ such that $B_{n} \cong A_{n}$ for all $n$. Note that each $\left[B_{n}\right]=\left[A_{n}\right]=x_{n}$. As $M_{t}(R)$ is right $\aleph_{0}$-continuous, $t R_{R}$ must have a finitely generated submodule $B$ such that $\cup B_{n} \leqq_{e} B$ [1, Proposition 14.3]. Set $x=[B]$; then each $x_{n} \leqq x$, and we claim that $x=\vee x_{n}$.

Thus consider any $y \in K_{0}(R)$ such that each $x_{n} \leqq y$. In particular, $y \geqq x_{1} \geqq 0$, hence $y=[C]$ for some finitely generated projective right $R$-module $C$. Then each $B_{n} \lesssim C$, whence Corollary 2.3 shows that $B \lesssim C$, and so $x \leqq y$.

Therefore $x=\vee x_{n}$, as desired.

COROLLARY 2.6. Let $R$ be a directly finite, right $\boldsymbol{\aleph}_{0}$-continuous, regular ring, let $A$ and $B$ be finitely generated projective right $R$-modules, and let $n \in \boldsymbol{N}$.

(a) If $n A \lesssim n B$, then $A \lesssim B$.

(b) If $n A \cong n B$, then $A \cong B$.

Proof. (a) In $K_{0}(R)$, we have $n[A] \leqq n[B]$. Since $K_{0}(R)$ is a monotone $\sigma$-complete interpolation group, [7, Corollary 1.4] implies that $[A] \leqq[B]$. Therefore $A \lesssim B$.

(b) Using (a), we obtain $A \lesssim B$ and $B \lesssim A$, whence $A \cong B$ by unit-regularity.

Corollary 2.6 generalizes [7, Corollary 2.2]. The upcoming corollaries generalize [7, Theorem 2.3].

DEFinition. We assume the reader is familiar with the concept of a pseudo-rank function on a regular ring $R$, and with the compact convex set $\boldsymbol{P}(R)$ of all pseudo-rank functions on $R$ [1, Chapters 16-18]. An extreme point of $\boldsymbol{P}(R)$ is any $P \in \boldsymbol{P}(R)$ which cannot be expressed as a nontrivial convex combination of other points of 
$\boldsymbol{P}(R)$, that is, whenever $P=\alpha P_{1}+(1-\alpha) P_{2}$ for some $P_{1}, P_{2} \in \boldsymbol{P}(R)$ and some real number $\alpha$ with $0 \leqq \alpha \leqq 1$, then $\alpha=0$, or $\alpha=1$, or $P_{1}=P_{2}=P$. We use $\partial_{e} \boldsymbol{P}(R)$ to denote the set of extreme points of $\boldsymbol{P}(R)$.

CoROllaRy 2.7. Let $R$ be a directly finite, right $\boldsymbol{\aleph}_{0}$-continuous, regular ring, let $A$ and $B$ be finitely generated projective right $R$ modules, and get

$$
A \cong x_{1} R \oplus \cdots \oplus x_{n} R ; \quad B \cong y_{1} R \oplus \cdots \oplus y_{k} R
$$

for some elements $x_{1}, \cdots, x_{n}, y_{1}, \cdots, y_{k} \in R$.

(a) $A \lesssim B$ if and only if $A / A M \lesssim B / B M$ for all maximal twosided ideals $M$ of $R$, if and only if

$$
P\left(x_{1}\right)+\cdots+P\left(x_{n}\right) \leqq P\left(y_{1}\right)+\cdots+P\left(y_{k}\right)
$$

for all $P \in \partial_{e} P(R)$.

(b) $A \cong B$ if and only if $A / A M \cong B / B M$ for all maximal twosided ideals $M$ of $R$, if and only if

$$
P\left(x_{1}\right)+\cdots+P\left(x_{n}\right)=P\left(y_{1}\right)+\cdots+P\left(y_{k}\right)
$$

for all $P \in \partial_{e} \boldsymbol{P}(R)$.

Proof. (a) If $A \lesssim B$, then obviously $A / A M \lesssim B / B M$ for all maximal two-sided ideals $M$ of $R$.

Now assume that $A / A M \lesssim B / B M$ for all maximal two-sided ideals $M$ of $R$. Let $P \in \partial_{e} P(R)$, set $M=\operatorname{ker}(P)$, and let $\bar{P}$ denote the rank function induced on $R / M$ by $P$. Then $R / M$ contains no uncountable direct sums of nonzero right ideals [1, Proposition 16.11], hence [1, Corollary 14.16] shows that $R / M$ is a continuous regular ring. In addition, $R / M$ is unit-regular, and by [1, Lemma 16.24] it is indecomposable. Consequently, [1, Corollary 13.20] shows that $R / M$ is right self-injective, hence we can infer from [1, Theorem 9.25] that $R / M$ is a simple ring.

Thus $M$ is a maximal two-sided ideal of $R$, whence $A / A M \lesssim$ $B / B M$. Using the notation $x \mapsto \bar{x}$ for the natural map $R \rightarrow R / M$, we obtain

$$
\begin{gathered}
\bar{x}_{1}(R / M) \oplus \cdots \oplus \bar{x}_{n}(R / M) \cong A / A M \lesssim B / B M \\
\cong \bar{y}_{1}(R / M) \oplus \cdots \oplus \bar{y}_{k}(R / M),
\end{gathered}
$$

and consequently $\Sigma P\left(x_{i}\right)=\Sigma \bar{P}\left(\bar{x}_{i}\right) \leqq \Sigma \bar{P}\left(\bar{y}_{j}\right)=\Sigma P\left(y_{j}\right)$, because of [1, Proposition 16.1].

Finally, assume that $\Sigma P\left(x_{i}\right) \leqq \Sigma P\left(y_{j}\right)$ for all $P \in \partial_{e} P(R)$. Because of the natural affine homeomorphism of the state space $S\left(K_{0}(R),[R]\right)$ 
onto $\boldsymbol{P}(R)$ [1, Proposition 17.12], it follows that

$$
s([A])=\Sigma s\left(\left[x_{i} R\right]\right)=\Sigma P\left(x_{i}\right) \leqq \Sigma P\left(y_{j}\right)=\Sigma s\left(\left[y_{j} R\right]\right)=s([B])
$$

for any $s \in \partial_{e} S\left(K_{0}(R),[R]\right)$, where $P$ denotes the pseudo-rank function in $\partial_{e} \boldsymbol{P}(R)$ corresponding to $s$. As $K_{0}(R)$ is a monotone $\sigma$-complete interpolation group, it is archimedean [7, Theorem 1.3], hence we conclude from [3, Proposition I.5.3] that $[A] \leqq[B]$. Therefore $A \lesssim B$.

(b) This is immediate from (a) and the unit-regularity of $R$.

CoROLlaRY 2.8. If $R$ is a directly finite, right $\boldsymbol{\aleph}_{0}$-continuous, regular ring, then $\operatorname{ker}(\boldsymbol{P}(R))=0$, and the intersection of the maximal two-sided ideals of $R$ is zero.

III. Countable interpolation. We show that all directly finite, right $\boldsymbol{\aleph}_{0}$-continuous, regular rings satisfy a certain countable interpolation property, from which further structural results follow.

Proposition 3.1. For a unit-regular ring $R$, the following properties are equivalent:

(a) If $A_{1}, A_{2}, \cdots$ and $B_{1}, B_{2}, \cdots$ are principal right ideals of $R$ such that $A_{i} \lesssim B_{j}$ for all $i, j$, then there exists a principal right ideal $C$ in $R$ such that $A_{i} \lesssim C \lesssim B_{j}$ for all $i, j$.

(b) If $A_{1}, A_{2}, \cdots$ and $B_{1}, B_{2}, \cdots$ are principal left ideals of $R$ such that $A_{i} \lesssim B_{j}$ for all $i, j$, then there exists a principal left ideal $C$ in $R$ such that $A_{i} \lesssim C \lesssim B_{j}$ for all $i, j$.

(c) If $x_{1}, x_{2}, \cdots$ and $y_{1}, y_{2}, \cdots$ are elements of $K_{0}(R)$ such that $x_{\imath} \leqq y_{j}$ for all $i, j$, then there exists $z \in K_{0}(R)$ such that $x_{i} \leqq z \leqq y_{j}$ for all $i, j$.

Proof. [3, Proposition II.12.1].

DEFINITION. We say that a unit-regular ring $R$ satisfies countable interpolation if the equivalent conditions of Proposition 3.1 hold. As shown in [3, Theorem II.12.3], all homomorphic images of $\aleph_{0}$-continuous regular rings satisfy countable interpolation. This also holds in our setting, as follows.

THEOREM 3.2. Let $R$ be a directly finite, right $\boldsymbol{\aleph}_{0}$-continuous, regular ring, and let $J$ be a two-sided ideal of $R$. Then $R / J$ is a unit-regular ring satisfying countable interpolation.

Proof. Since $K_{0}(R)$ is a monotone $\sigma$-complete interpolation group (Theorem 2.5), [7, Lemma 1.2] shows that $K_{0}(R)$ has countable inter- 
polation. Thus $R$ is a unit-regular ring with countable interpolation. By [3, Proposition II.12.2], these properties carry over to $R / J$.

As a good portion of the structure theory for $\boldsymbol{\aleph}_{0}$-continuous regular rings developed in [3] was developed in terms of unit-regular rings with countable interpolation, it now carries over to directly finite, right $\aleph_{0}$-continuous, regular rings. For the complete theory, we refer the reader to [3, Chapters II.12-II.15]; here we shall only sketch some of the highlights.

Definition. We use Max $\operatorname{Spec}(R)$ to denote the collection of all maximal two-sided ideals in a ring $R$, equipped with the usual hull-kernel topology.

Proposition 3.3. Let $R$ be a directly finite, right $\boldsymbol{\aleph}_{0}$-continuous, regular ring.

(a) There is a continuous bijection $\theta: \partial_{e} P(R) \rightarrow \operatorname{Max} \operatorname{Spec}(R)$ given by the rule $\theta(N)=\operatorname{ker}(N)$.

(b) $\theta$ maps compact subsets of $\partial_{e} \boldsymbol{P}(R)$ onto closed subsets of $\operatorname{Max} \operatorname{Spec}(R)$.

(c) $\theta$ is a homeomorphism if and only if $\partial_{e} \boldsymbol{P}(R)$ is compact, if and only if $\operatorname{Max} \operatorname{Spec}(R)$ is Hausdorff.

Proof. [3, Proposition II.14.5].

Definition. A regular ring $R$ is said to satisfy general comparability provided that for any $x, y \in R$, there exists a central idempotent $e \in R$ such that $e x R \lesssim e y R$ and $(1-e) y R \lesssim(1-e) x R$.

THEOREM 3.4. Let $R$ be a directly finite, right $\boldsymbol{\aleph}_{0}$-continuous, regular ring. Then the following conditions are equivalent:

(a) $R$ satisfies general comparability.

(b) $\partial_{e} P(R)$ is compact.

(c) $\operatorname{Max} \operatorname{Spec}(R)$ is Hausdorff.

(d) $K_{0}(R)$ is a lattice.

Proof. This is proved for $\aleph_{0}$-continuous regular rings in [3, Theorem II.14.9]. The same proof works here, because $\operatorname{ker}(\boldsymbol{P}(R))=$ 0 (Corollary 2.8) and $K_{0}(R)$ is a monotone $\sigma$-complete interpolation group (Theorem 2.5).

THEOREM 3.5. Let $R$ be a directly finite, right $\boldsymbol{\aleph}_{0}$-continuous, regular ring, and let $J$ be a two-sided ideal of $R$. Then $R / J$ is right $\aleph_{0}$-continuous if and only if $K_{0}(R / J)$ is monotone $\sigma$-complete. 
Proof. This is proved in [3, Theorem II.14.12] for $\boldsymbol{\aleph}_{0}$-continuous regular rings. By using only the right-sided portion of that proof, we obtain the desired result here.

Definition. Given a compact convex set $S$, we use Aff $(S)$ to denote the partially ordered real Banach space of all affine continuous real-valued functions on $S$ (with the pointwise ordering and the supremum norm).

Definition. For any regular ring $R$, there is a natural positive group homomorphism

$$
\varphi: K_{0}(R) \longrightarrow \text { Aff }(P(R))
$$

such that $\varphi([x R])(N)=N(x)$ for all $x \in R$ and all $N \in P(R)$ [3, p. 120]. We refer to $\varphi$ as the natural map from $K_{0}(R)$ to $\operatorname{Aff}(\boldsymbol{P}(R))$.

THEOREM 3.6. Let $R$ be a directly finite, right $\boldsymbol{\aleph}_{0}$-continuous, regular ring. For any $N \in \partial_{e} \boldsymbol{P}(R)$ such that $R / \operatorname{ker}(N)$ is isomorphic to an $n \times n$ matrix ring over a division ring, set $A_{N}=(1 / n) Z$; for all other $N \in \partial_{e} \boldsymbol{P}(R)$, set $A_{N}=\boldsymbol{R}$. Set

$$
A=\left\{p \in \operatorname{Aff}(\boldsymbol{P}(R)) \mid p(N) \in A_{N} \text { for all } N \in \partial_{e} \boldsymbol{P}(R)\right\} .
$$

Then the natural map $\varphi: K_{0}(R) \rightarrow \operatorname{Aff}(P(R))$ provides an isomorphism of $\left(K_{0}(R),[R]\right)$ onto $(A, 1)$ (as partially ordered abelian groups with order-unit).

Proof. [3, Theorem II.15.1].

CoRollary 3.7. Let $R$ be a directly finite, right $\boldsymbol{\aleph}_{0}$-continuous, regular ring, and let $t \in N$. If every simple artinian homomorphic image of $R$ is a $t \times t$ matrix ring, then $R$ is a $t \times t$ matrix ring.

Proof. This follows from Theorem 3.6 as [3, Theorem II.15.3] follows from [3, Theorem II.15.1]. (The statement of [3, Theorem II.15.3] includes the hypothesis that all simple homomorphic images of $R$ be $t \times t$ matrix rings; however, an examination of the proof shows that it suffices to assume this just for simple artinian homomorphic images.)

Corollary 3.8. Let $R$ be a directly finite, right $\boldsymbol{\aleph}_{0}$-continuous, regular ring with no simple artinian homomorphic images. Then $R$ is a $t \times t$ matrix ring for every $t \in N$. Moreover, given $t \in N$ and any finitely generated projective right $R$-module $B$, there exists a 
finitely generated projective right $R$-module $C$ such that $B \cong t C$.

Proof. [3, Theorem II.15.4].

A number of the results of this paper, such as Corollaries 2.6, 2.7, 2.8, Proposition 3.3, Theorem 3.6, and Corollaries 3.7 and 3.8, also hold for $N^{*}$-complete regular rings, as proved in [2]. The $\boldsymbol{\aleph}_{0}$ continuous cases of these results may be regarded as corollaries of the $N^{*}$-complete results, since every $\boldsymbol{\aleph}_{0}$-continuous regular ring is $N^{*}$-complete [2, Theorem 2.3]. However, it is an open problem whether directly finite, right $\boldsymbol{\aleph}_{0}$-continuous, regular rings must be $N^{*}$-complete (we conjecture that they need not be), so the results of this paper are not corollaries of the $N^{*}$-complete theory.

\section{REFERENCES}

1. K. R. Goodearl, Von Neumann Regular Rings, London (1979), Pitman.

2. - Metrically complete regular rings, Trans. Amer. Math. Soc., (to appear).

3. K. R. Goodearl, D. E. Handelman, and J. W. Lawrence, Affine representations of Grothendieck groups and applications to Rickart $C^{*}$-algebras and $\boldsymbol{\aleph}_{0}$-continuous regular rings, Memoirs Amer. Math. Soc., No. 234 (1980).

4. I. Halperin, On the transitivity of perspectivity in continuous geometries, Trans. Amer. Math. Soc., 44 (1938), 537-562.

5. D. Handelman, Perspectivity and cancellation in regular rings, J. Algebra, 48 (1977), 1-16.

6. - Finite Rickart $C^{*}$-algebras and their properties, in Studies in Analysis, Advances in Math. Suppl. Studies, Vol. 4 (1979), 171-196.

7. D. Handelman, D. Higgs, and J. Lawrence, Directed abelian groups, countably continuous rings, and Rickart $C^{*}$-algebras, J. London Math. Soc., (2) 21 (1980), 193-202.

Received January 11, 1981. This research was partially supported by a grant from the National Science Foundation.

UNIVERSITY OF UTAH

Salt Lake City, UT 84112 


\section{PACIFIC JOURNAL OF MATHEMATICS}

\section{EDITORS}

DONALD BABBITT (Managing Editor)

University of California

Los Angeles, California 90024

Hugo RossI

University of Utah

Salt Lake City, UT 84112

C. C. MOORE and ARTHUR Agus

University of California

Berkeley, CA 94720

\section{J. DUGUNDJI}

Department of Mathematics University of Southern California Los Angeles, California 90007

R. FinN and J. MILGRAM Stanford University Stanford, California 94305

\section{ASSOCIATE EDITORS}
R. ARNES
E. F. BeCKENBACH
B. H. Neumann
F. WOLF
K. YosHIDA

\section{SUPPORTING INSTITUTIONS}

UNIVERSITY OF ARIZONA

UNIVERSITY OF BRITISH COLUMBIA

CALIFORNIA INSTITUTE OF TECHNOLOGY

UNIVERSITY OF CALIFORNIA

MONTANA STATE UNIVERSITY

UNIVERSITY OF NEVADA, RENO

NEW MEXICO STATE UNIVERSITY

OREGON STATE UNIVERSITY
UNIVERSITY OF OREGON UNIVERSITY OF SOUTHERN CALIFORNIA STANFORD UNIVERSITY UNIVERSITY OF HAWAII UNIVERSITY OF TOKYO UNIVERSITY OF UTAH WASHINGTON STATE UNIVERSITY UNIVERSITY OF WASHINGTON 


\section{Pacific Journal of Mathematics}

Vol. 100, No. $1 \quad$ September, 1982

Charalambos D. Aliprantis, Owen Sidney Burkinshaw and M. Duhoux,

Compactness properties of abstract kernel operators $\ldots \ldots \ldots \ldots \ldots \ldots 1$

Roger C. Alperin, Locally compact groups acting on trees .............23

Robert F. Brown, Real homology of Lie group homomorphisms ......... 33

Karen Chase, Maximal groups in sandwich semigroups of binary relations . . 43

W. Wistar (William) Comfort and T. Soundararajan, Pseudocompact

group topologies and totally dense subgroups $\ldots \ldots \ldots \ldots \ldots \ldots \ldots 61$

M. Ferri and C. Gagliardi, Crystallisation moves $\ldots \ldots \ldots \ldots \ldots \ldots \ldots$

Kenneth R. Goodearl, Directly finite aleph-nought-continuous regular

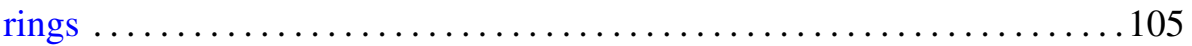

Edward Lewis Green, On the representation theory of rings in matrix

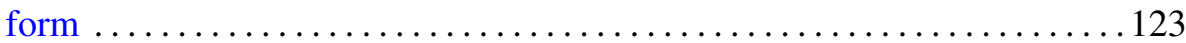

Walter Hengartner and Glenn E. Schober, Interpolation, continuation, and

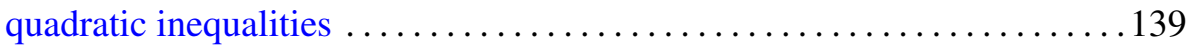

Kenneth Kunen and Haskell Paul Rosenthal, Martingale proofs of some geometrical results in Banach space theory $\ldots \ldots \ldots \ldots \ldots \ldots \ldots \ldots \ldots \ldots$

Brian William McEnnis, Shifts on indefinite inner product spaces. II . . . . . 177

Roman Pol, Note on the spaces $P(S)$ of regular probability measures whose topology is determined by countable subsets $\ldots \ldots \ldots \ldots \ldots \ldots \ldots \ldots 185$

Joan Manuel Verdera Melenchón, Finitely generated projective extensions

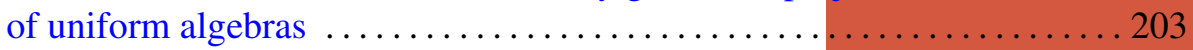

Cheng Ye You, Fixed point classes of a fiber map .................. 217 